\title{
TPPP3 Associated with Prognosis and Immune Infiltrates in Head and Neck Squamous Carcinoma
}

\author{
Zheng Yang, ${ }^{1}$ Xiaohong Li, ${ }^{1}$ Jingyu Li, ${ }^{2}$ Qisheng Su, ${ }^{1}$ Yuling Qiu, ${ }^{1}$ Zunni Zhang, \\ Liqian Zhang, ${ }^{1}$ and Wuning Mo ${ }^{1}$ \\ ${ }^{1}$ Department of Clinical Laboratory, First Affiliated Hospital Guangxi Medical University, Nanning, \\ Guangxi Zhuang Autonomous Region, China \\ ${ }^{2}$ Department of Otorhinolaryngology and Head and Neck Surgery, First Affiliated Hospital Guangxi Medical University, Nanning, \\ Guangxi Zhuang Autonomous Region, China
}

Correspondence should be addressed to Wuning Mo; mown16300@126.com

Received 26 May 2020; Revised 8 September 2020; Accepted 16 September 2020; Published 7 October 2020

Academic Editor: Bilal Alatas

Copyright (c) 2020 Zheng Yang et al. This is an open access article distributed under the Creative Commons Attribution License, which permits unrestricted use, distribution, and reproduction in any medium, provided the original work is properly cited.

Tubulin polymerization promoting protein family member 3 (TPPP3) is a kind of protein that can mediate the dynamics and stability of microtubules. However, the correlations of TPPP3 between prognosis and immune infiltrates in different tumors are still unclear. The analysis of TPPP3 expression was performed via Oncomine and Gene Expression Profiling Interactive Analysis (GEPIA) website. We also used GEPIA to assess the impact of TPPPT3 on clinical outcomes. The related pathways involved in TPPP3 were analyzed by gene-set enrichment analysis (GSEA), and the correlation between TPPP3 and immune infiltration was studied by Tumor Immune Estimation Resource2.0 (TIMER 2.0). The TPPP3 expression was significantly reduced in head and neck squamous carcinoma (HNSC) compared to adjacent tissues. In addition, the low expression of TPPP3 in HNSC was significantly associated with prognosis. The pathways closely related to the low expression of TPPP3 are "Antigen Processing and Presentation," "Primary Immunodeficiency," and so on. The TPPP3 expression was negatively correlated with the level of CD8+ T cell, B cell, and myeloid dendritic cell infiltration in HNSC. The TPPP3 expression is closely related to multiple immunomarkers in CD8+ T cell and Myeloid dendritic cells. These data indicate that TPPP3 is associated with multiple cancers and involves multiple immune-related pathways, and TPPP3 is associated with immune infiltration levels. Besides, the TPPP3 expression may help regulate tumor-associated CD8 + T cells, DC cells in HNSC. We conclude TPPP3 can be considered as a biomarker for predicting head and neck squamous cell carcinoma prognosis and immune infiltration.

\section{Introduction}

Head and neck squamous carcinoma (HNSC) is one of the most frequent tumors in Southeast Asia and southern China. The mechanism of HNSC development is complex and involves the alteration of polygenic and multisignaling pathways [1]. During this process, viral factors, environmental factors, and genetic factors affect tumor-related gene regulation and abnormal expression [2-5]. The early diagnosis of HNSC is difficult due to the hidden physiological position of HNSC, and it is prone to lymph node and distant metastasis. Therefore, screening biomarkers are helpful for the diagnosis and pathological indicators of tumorigenesis and development.
TPPP3, also known as TPPP/p20, is a protein-coding gene located on chromosome 16 that was first reported in 2006. ТPPP3 is a brain-specific protein homologous to $\mathrm{TPPP} / \mathrm{p} 25$, expressed in many human cells and organs, which could induce tubulin polymerization and microtubule (MT) bundling [6]. Microtubules are the main components of mitotic spindles, which control all aspects of cell division and chromosome separation. Studies have shown that microtubule dynamics change in cancer cell division and are associated with the development of chromosomal instability, anaplasia, and drug resistance [7]. Many kinds of tumor drugs exert their anticancer effect by acting on microtubules and microtubule-related proteins. The classical anticancer drugs such as vincristine and paclitaxel are widely used in 
the clinic, so the further exploration of microtubules and tubulin is of great significance to the study of tumor prevention and treatment.

A growing number of researchers in recent years have begun to explore the relationship between TPPP3 and tumors. Studies suggest that reduced TPPP 3 can lead to abnormal mitoses, such as the formation of multipolar spindles and chromosome segregation errors, leading to HeLa apoptosis [8]. However, the potential role of TPPP3 in HNSC development or metastasis remains unknown.

In the tumor microenvironment, immune and stromal cells are two major types of nontumor components. The extent of tumor immune infiltration and stromal cells has significant value for tumor diagnosis and prognosis evaluation. This study provides a comprehensive analysis of TPPP 3 expression in cancer databases and its relationship to the prognosis of cancer patients. Then, we performed pathological verification with clinical specimens. In addition, we also examined the relationship between TPPP3 and tumorinfiltrating immune cells in HNSC through the Tumor Immunity Estimation Resource 2.0(TIMER 2.0). This report clarifies the important role of TPPP 3 in HNSC and provides evidence for the relationship between TPPP 3 and tumorimmune cell infiltration interactions.

\section{Results}

2.1. TPPP3 mRNA Expression Levels in Different Types of Human Cancer. To understand the differences in TPPP 3 expression between human tumor and nontumor tissues, we utilized the Oncomine database to analyze the expression levels of TPPP 3 in multiple cancer types and different tumors and normal tissues. Comparing to normal tissues, the study demonstrated that TPPP 3 observed lower expression in bladder cancer, brain cancer, breast cancer, head and neck cancer, kidney cancer, lung cancer, melanoma, ovarian cancer, and sarcoma and high expression in gastric cancer (Figure 1(a)). To more accurately assess TPPP 3 expression in human cancers, we used RNA-seq data from 31 malignancies in The Cancer Genome Atlas (TCGA) and Genotype-Tissue Expression (GTEx) to examine the TPPP3 expression. Figure 1(b) shows the differential expression of TPPP 3 between tumors and normal tissues. The TPPP3 expression was significantly decreased in HNSC, bladder urothelial carcinoma (BLCA), breast invasive carcinoma (BRCA), kidney chromophobe (KICH), kidney renal papillary cell carcinoma (KIPC), lung adenocarcinoma (LUAD), and lung squamous cell carcinoma (LUSC). However, compared with adjacent normal tissues, the TPPP 3 expression was significantly higher in cholangiocarcinoma (CHOL) and kidney renal clear cell carcinoma (KIRC).

2.2. Prognostic Potential of TPPP3 in Cancers. To understand the prognosis of TPPP3 in tumors, Gene Expression Profiling Interactive Analysis (GEPIA) was used to determine whether TPPP3 expression is correlated with the prognosis of HNSC, LUAD, LUSC, KICH, BRCA, KIRC, and BLCA. (Figures 2(a)-2(g)) Notably, the low expression of TPPP3 affects the prognosis of HNSC $(p=0.027, \mathrm{HR}=0.74)$.
2.3. TPPP3 Gene Set Enrichment Analyses. To discover the underlying mechanism of TPPP3 and HNSC, Gene Set Enrichment Analysis (GSEA) was utilized to obtain the TPPP3-related gene collection based on the actual overall trend analysis and compare enrichment analysis such as Kyoto Encyclopedia of Genes and Genomes (KEGG) and Gene Ontology (GO). Consequently, the enrichment of 100 functional gene sets was obtained. The pathways closely related to the low expression of TPPP3 are "Antigen Processing and Presentation," "Primary Immunodeficiency," "RIG I Like Receptor Signaling Pathway," "Endometrial Cancer," and "P53 Signaling Pathway." The pathways associated with TPPP3 high expression are "Parkingson Disease," "Oxidative Phosphorylation," and "Phenylalanine Metabolism" (Figures 2(h)-2(o)). "Translation repressor activity mRNA regulatory element-binding," "Negative regulation of regulated secretory pathway," "Negative regulation of regulated secretory pathway," "Amino acid betaine metabolic process," "Protein kinase a regulatory subunit binding," and "Site of DNA damage" are the six most enriched items in GO related to the low expression of TPPP3 (Figures 2(p)-2(t)). The GO items associated with TPPP3 high expression are "Nuclear envelope reassembly," "High density lipoprotein particle clearance," "High density lipoprotein particle remodeling," "Reverse cholesterol transport," and "Negative regulation of lipase activity" (Figures 2(u)-2(y)).

2.4. TPPP3 Expression Is Associated with Immune Infiltration Levels in HNSC. Tumor-infiltrating lymphocytes are independent risk factors affecting tumor prognosis [9]. Therefore, we analyzed by TIMER2.0 whether the TPPP3 expression was associated with immune infiltration levels in HNSC. The results show that the TPPP 3 expression has a certain correlation with the infiltration of $\mathrm{CD} 8+\mathrm{T}$ cells, $\mathrm{B}$ cells, and myeloid dendritic cells (Figure 3).

2.5. Analysis of the Correlation between TPPP3 Expression and Immune Cell Genes. To gain insight into the intrinsic link between TPPP 3 and related immune cells, we applied the TIMER 2.0 database to analyze the correlation between TPPP3 genes in HNSC related immune infiltrating cells. We obtained results on the correlation between TPPP 3 expression and marker genes of tumor infiltrationassociated immune cells, including $\mathrm{CD} 8+\mathrm{T}$ cells (CD8A and CD8B), B cell (CD19 and CD79a), and Myeloid dendritic cell (HLA-DPB1, HLA-DQB1, HLA-DRA, HLA-DPA1, CD1C, NRP1, and ITGAX). The results manifested that TPPP3 expression levels were negatively correlated with most of the immune marker genes in HNSC (Figure 4). In the high-level immune infiltration mode of HNSC, TPPP3 has an intense correlation with CD8 $+\mathrm{T}$ cell marker and DC markers. These results further indicate that there is a certain relationship between TPPP3 and DC penetration. Promote dendritic cells to regulate cytotoxic CD8 + T cell responses and exert anticancer effects $[10,11]$. Whether TPPP3 is a key factor in mediating CD8 $+\mathrm{T}$ cell, DC, and tumor metastasis need further research. 


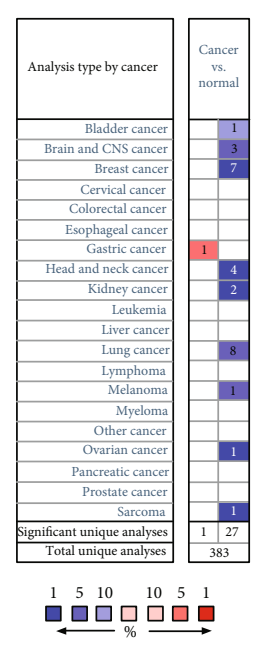

(a)

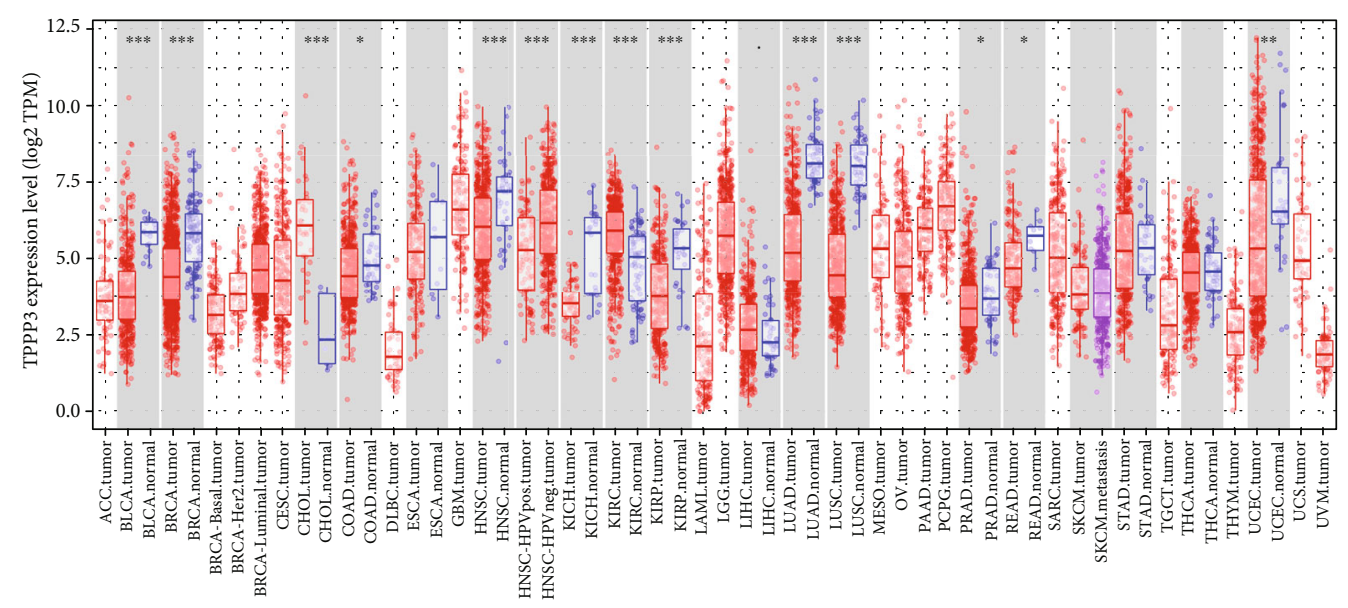

(b)

FIGURE 1: TPPP3 expression levels in different types of human tumors. (a) In the Oncomine database, TPPP3 expression in different cancer datasets compared to normal tissues. (b) Human TPPP3 expression levels in different tumor types from Timer. BRCA (breast invasive carcinoma), CESC (cervical squamous cell carcinoma and endocervical adenocarcinoma), CHOL (cholangiocarcinoma), COAD (colon adenocarcinoma), DLBC (Lymphoid Neoplasm Diffuse Large B-cell Lymphoma), ESCA (Esophageal carcinoma), GBM (Glioblastoma multiforme), HNSC (head and neck squamous cell carcinoma), KICH (kidney chromophobe), KIRC (kidney renal clear cell carcinoma), KIRP (kidney renal papillary cell carcinoma), LAML (Acute Myeloid Leukemia), LGG (Brain Lower Grade Glioma), LIHC (Liver hepatocellular carcinoma), LUAD (lung adenocarcinoma), LUSC (lung squamous cell carcinoma), MESO (Mesothelioma), OV (ovarian serous cystadenocarcinoma), PAAD (Pancreatic adenocarcinoma), PCPG (Pheochromocytoma and Paraganglioma), PRAD (Prostate adenocarcinoma), READ (Rectum adenocarcinoma), SARC (sarcoma), SKCM (Skin Cutaneous Melanoma), STAD (Stomach adenocarcinoma), TGCT (Testicular Germ Cell Tumors), THCA (Thyroid carcinoma), THYM (Thymoma), UCEC (Uterine Corpus Endometrial Carcinoma), UCS (Uterine Carcinosarcoma), and UVM (Uveal Melanoma).

2.6. Low Expression of TPPP3 in Clinical Specimens of NPC. In the present research, the expression of TPPP3 in nasopharyngeal carcinoma (NPC) tissues was detected in 57 cases of NPC and 30 cases of normal tissue by immunohistochemistry, and the relationship between TPPP 3 and clinicopathological features of NPC was analyzed at the same time. The results showed that the expression of TPPP3 in NPC was significantly lower than that in normal nasopharyngeal tissue; the difference was statistically significant (Figure 5, Table 1). But there was no significant correlation with clinicopathological features (Table 2).

\section{Discussion}

A growing number of researchers have been exploring the relationship between TPPP3 and tumor development in recent years. According to the Zhou et al.'s study, knockdown of TPPP3 inhibits cell proliferation of HeLa cells while inducing cell cycle arrest [8]. Li et al. demonstrated that TPPP3 was highly expressed in nonsmall cell lung cancer, and that the high expression of TPPP3 was positively correlated with clinical stage, tumor volume, lymph node metastasis, and poor prognosis [12]. Ye et al. also revealed that TPPP3 was highly expressed in colorectal cancer and was associated with colorectal cancer progression and poor prognosis, and that interfering with TPPP3 expression suppressed tumor cell proliferation, migration, and invasion, and increased apoptosis [13]. Although TPPP3 has not been extensively studied, according to the available data, it is found that the expression level of TPPP3 was increased in some tumors and correlated with tumor proliferation. The expression of TPPP3 is upregulated in many tumors, and experimental studies have confirmed its oncogenic effect. We carried out a pan-cancer analysis of TPPP3, which indicates that TPPP3 has a different expression trend with other tumors in HNSC. However, no relevant studies were found in head and neck squamous carcinoma. Therefore, it is necessary to carry out detailed research on TPPP3 in HNSC.

In this article, we first performed a pan-cancer analysis of TPPP3 expression. The Oncomine database and GEPIA were used to analyze the mRNA expression data of 31 tumors to check the expression level and prognosis of TPPP 3 in different types of tumors. According to the results of the Oncomine database, we found that TPPP 3 was lower expressed in breast cancer, head and neck cancer, kidney cancer, ovarian cancer, and sarcoma compared to normal tissues, while higher expression in gastric cancer. Furthermore, GEPIA demonstrated that compared with neighboring normal tissues, the expression of TPPP3 in HNSC, BLCA, BRCA, KICH, KIRC, LUAD, and LUSC was significantly reduced, while the expression of TPPP3 in CHOL and KIRC was significantly higher. We found that using different databases, TPPP3 expression levels 


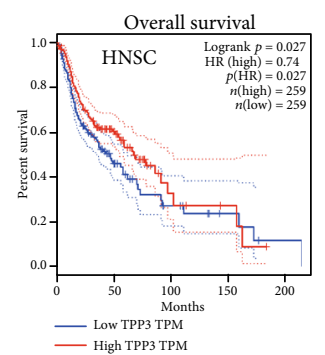

(a)

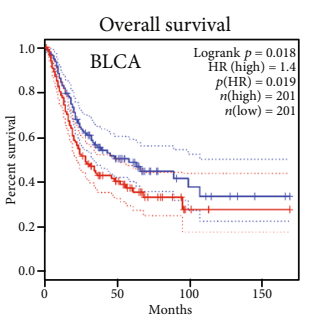

${ }_{\text {— }}^{\text {Ligh TPP3 } 1 \text { TPM }}$

(f)

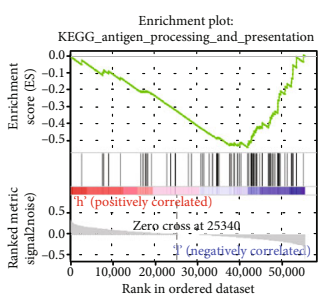

(k)

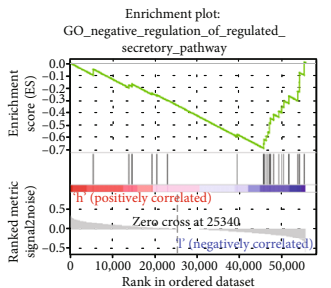

(p)

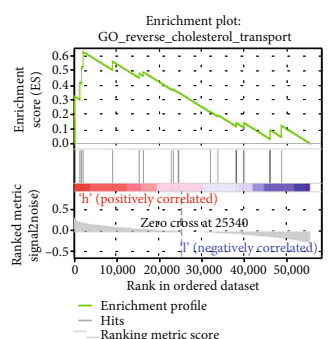

(u)

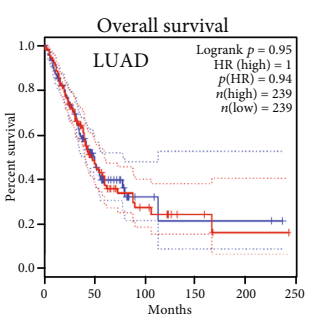

(b)

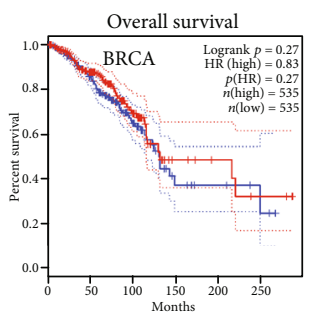

(g)

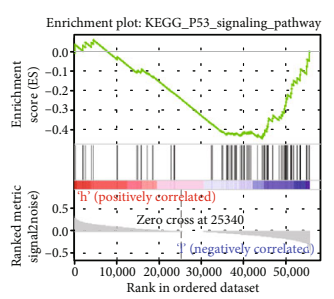

(l)

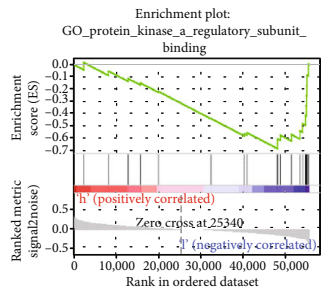

(q)

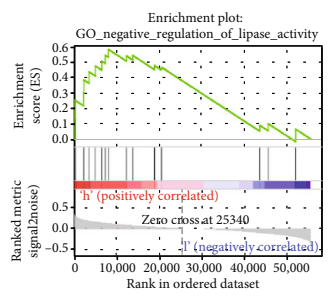

(v)

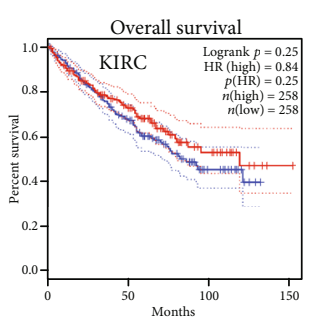

(c)

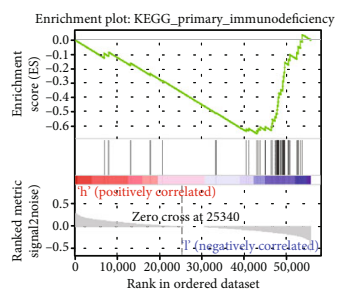

(h)

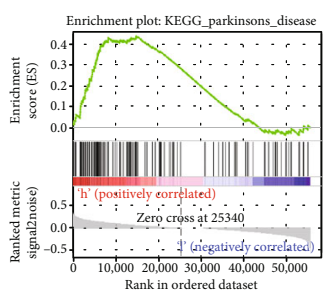

(m)

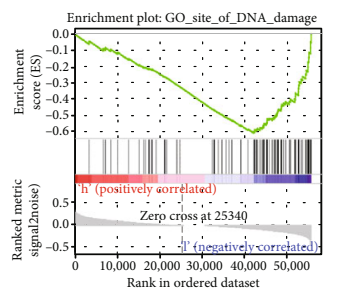

(r)

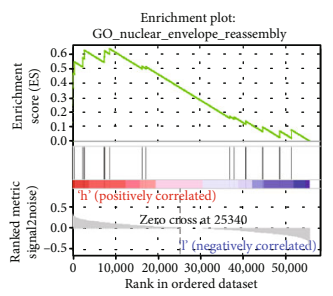

(w)

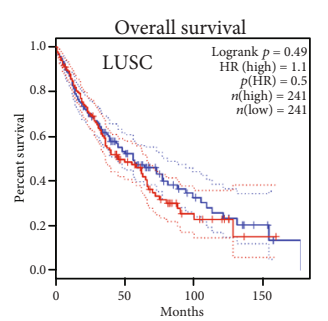

(d)

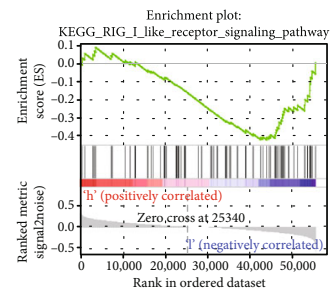

(i)

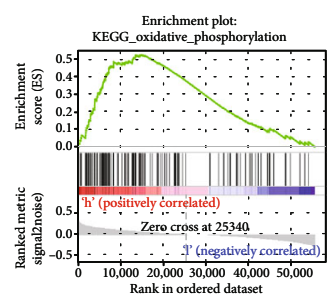

(n)

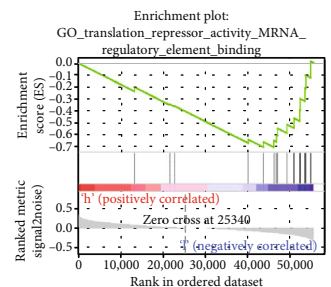

(s)

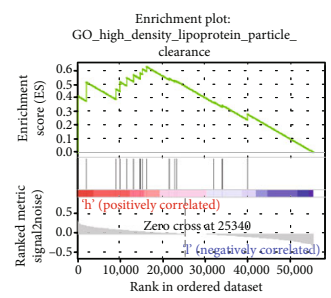

(x)

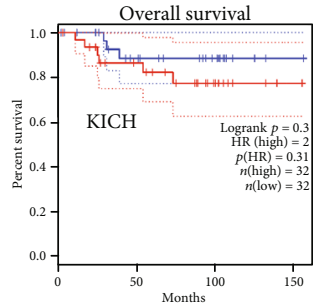

(e)

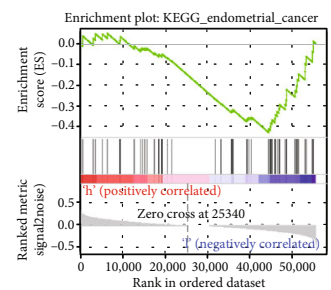

(j)

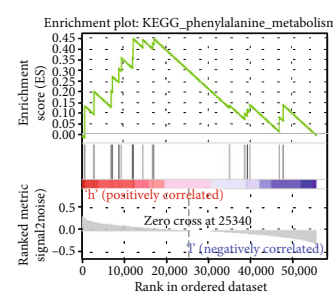

(o)

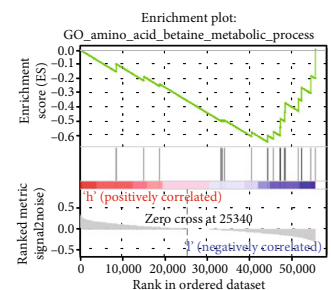

(t)

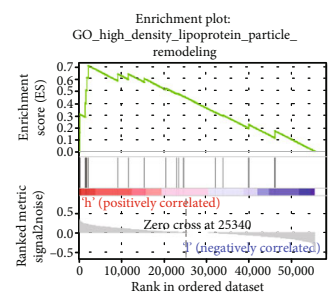

(y)

FIgURe 2: (a-g) Kaplan-Meier survival curves comparing the high and low expression of TPPP3 in different types of tumors by GEPIA. (h-y) list the most common functional gene sets enriched in HNSC samples with low and high expression of TPPP3.

differed among different tumor types, which may be due to the data collection method and the different biological characteristics of TPPP3. Interestingly, in these databases, we found consistency in TPPP3 expression in head and neck squamous cell carcinoma. In addition, the analysis of survival data indicates that low levels of TPPP3 expression are associated with a poor prognosis for HNSC.
In this research on the mechanism of TPPP3, we selected the transcript data of 70 cases HNSC in the TCGA database and performed GSEA analysis on these datasets. The pathways closely related to the low expression of TPPP 3 are "Antigen Processing and Presentation," "Primary Immunodeficiency," "RIG I Like Receptor Signaling Pathway," "Endometrial Cancer," and "P53 Signaling Pathway." Results 

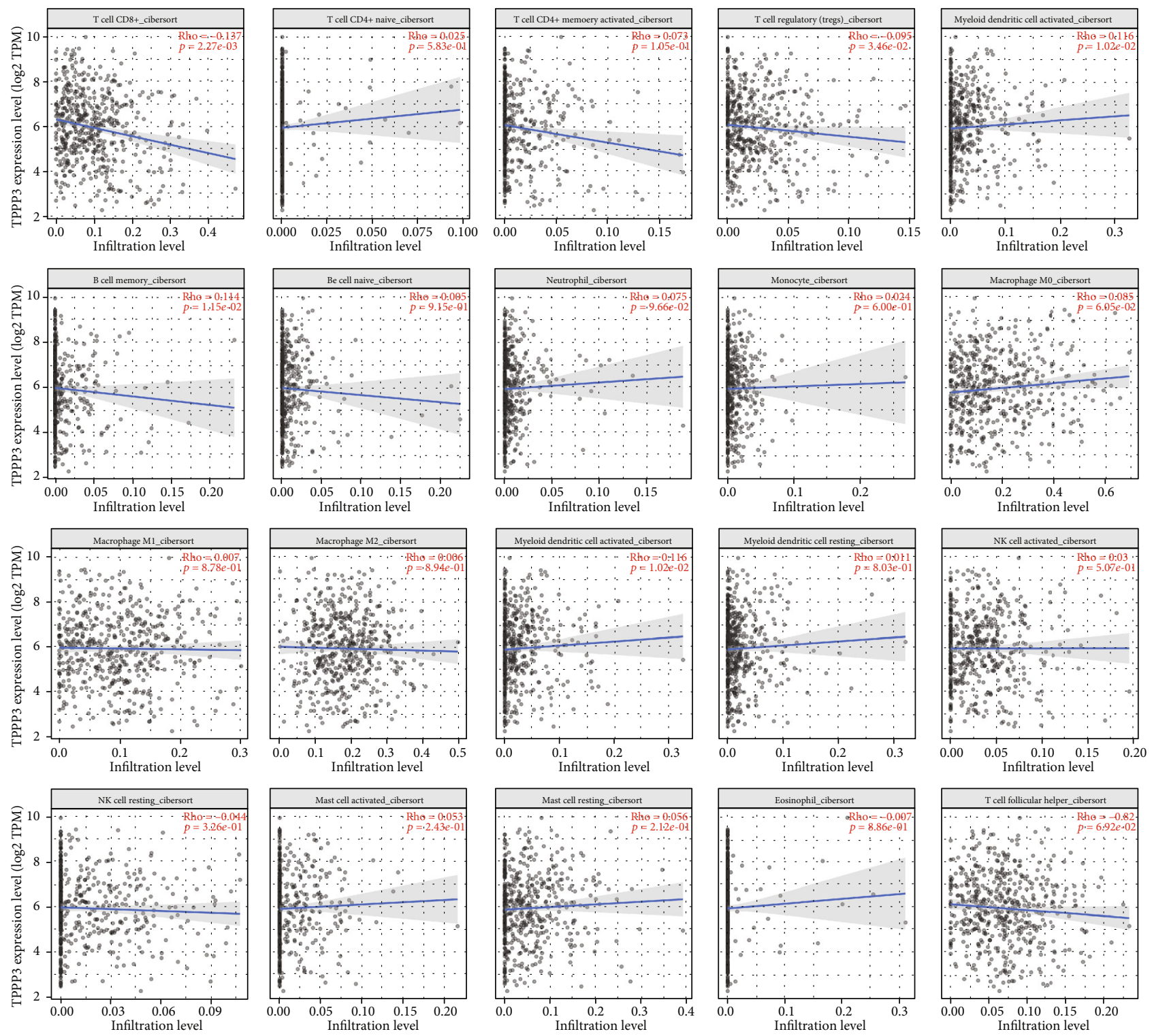

FIGURE 3: The correlation of TPPP3 expression with immune infiltration level in HNSC. TPPP3 expression is negatively correlated with infiltrating levels of CD8+ T cell, B cell, and myeloid dendritic cell. The infiltration level for each SCNA category is compared with the normal using a two-sided Wilcoxon rank-sum test.

also indicate that TPPP3 may be related to the mechanism of tumor immunity. Therefore, we shifted the research focus of TPPP3 to research on tumor immune infiltration. Our results indicate that TPPP3 expression is correlated with multiple immune cell infiltration levels in head and neck squamous cell carcinoma. There is a negative correlation between the expression of TPPP3 and the infiltration levels of CD8+T cells and B memory cells. However, TPPP 3 expression is positively correlated with DC cells. Moreover, the correlation between TPPP 3 expression and immune cell marker genes suggests that TPPP3 is regulating the immunological role of HNSC. Further, the genetic markers of CD8+ T cells and myeloid dendritic cells are weakly correlated with TPPP3 expression. These results reveal a potential regulatory role of TPPP3 in tumor-associated myeloid dendritic cellmediated $\mathrm{T}$ cell toxic effects.
In addition, to further understanding of TPPP3 expression in head and neck tumors, we also performed immunohistochemistry on clinical NPC specimens and validated tumor and normal tissue TPPP3 mRNA expression in two GEO NPC datasets. The results we obtained are consistent with those of Oncomine and GEPIA in that TPPP3 expression is significantly reduced in the HNSC. But there is no significant correlation with clinicopathological features. This study for the first time analyzed the expression and prognosis of TPPP3 in head and neck squamous cell carcinoma, unexpectedly, in contrast to the expression in other tumors. We are as well the first to reveal the link between TPPP 3 and immune infiltration. This study also has some shortcomings, such as we still need to perform in vitro and in vivo experiments on TPPP 3 for cellular function studies as well as pathway studies. 

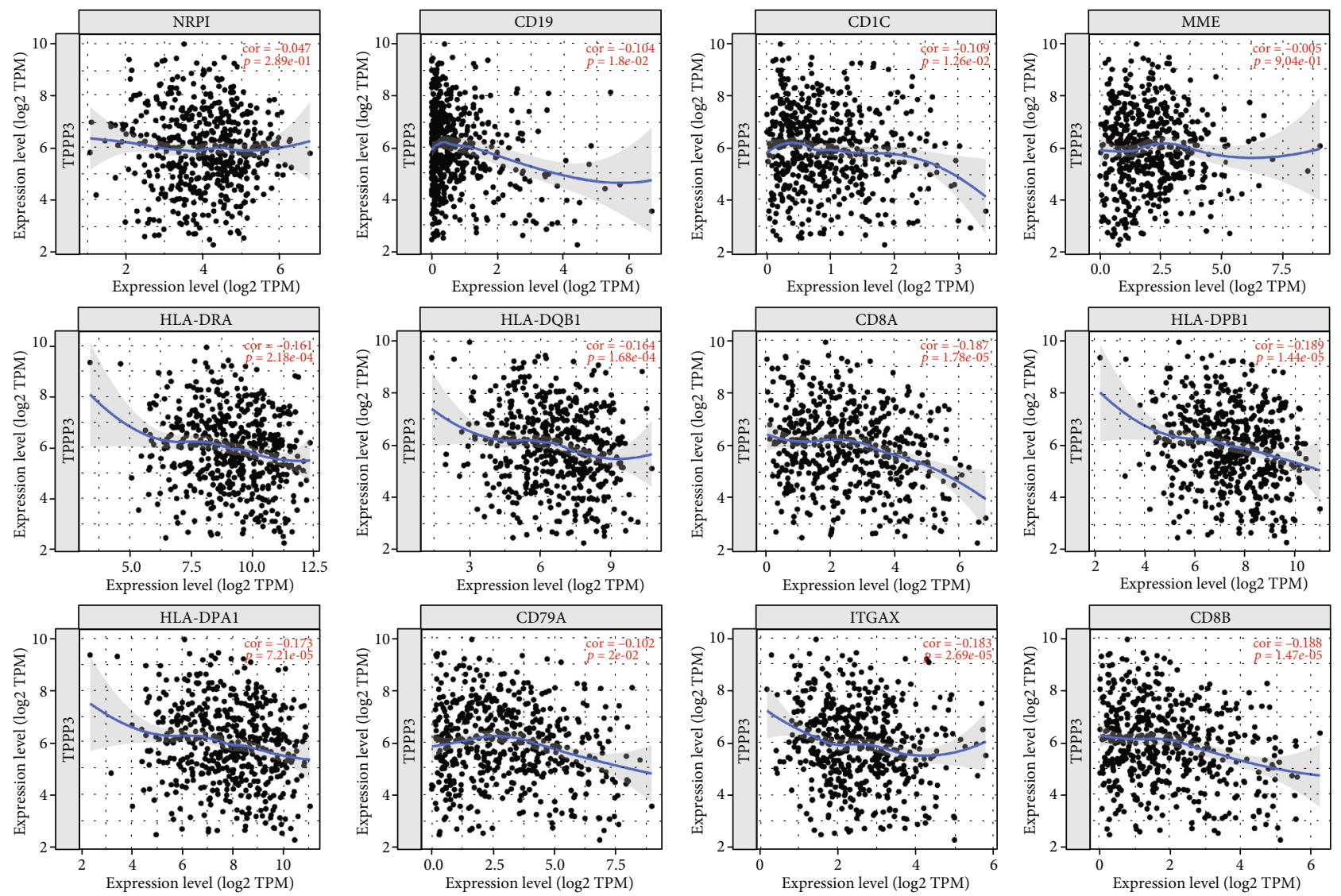

FIGURE 4: Scatterplots of correlations between TPPP3 expression and gene markers of CD8+T cells, B cell, and myeloid dendritic cell.

\section{Materials and Methods}

4.1. Clinical Specimens. NPC tissues from 57 confirmed and untreated NPC patients (median age: 45 years old; female: $n=16$; male: $n=41$ ) were collected from the First Affiliated Hospital of Guangxi Medical University in 2016-2019. The diagnoses were according to the World Health Organization (WHO) classification. And 30 nontumor nasopharyngeal tissues obtained by fiber optic nasopharyngoscopy and tonsillectomy were used as controls. Informed consent was obtained from all donors involved, and ethical approval for this study was granted by the Ethical Review Committee of the First Affiliated Hospital of Guangxi Medical University.

4.2. Oncomine Database Analysis. Oncomine (https://www .oncomine.org/resource/login.html) [14] is the world's largest cancer gene microarray database and integrated data-mining platform, which can be used to analyze gene expression differences, search for outliers, and predict coexpression genes. The Oncomine database was utilized to determine the expression level of the TPPP3 gene in various types of cancer. Threshold determinations were set as follows: $p$ value of 0.001 , fold change of 1.5 , and gene ranking of all.

4.3. GEPIA Website Analysis. GEPIA (http://gepia.cancerpku.cn/index.html) [15] is a newly created interactive web server for analyzing RNA sequencing expression data of tumor and normal samples from the TCGA and GTEx projects. GEPIA was employed to analyze the expression of the TPPP3 gene in 33 different types of tumors and generate survival curves including overall survival (OS) and disease-free survival (DFS). The significantly related genes were further confirmed by GEPIA, and the correlation coefficient was determined by Spearman's method.

4.4. TCGA Database. TCGA is the world's largest cancer database, which includes clinical data, genomic variation, mRNA expression, miRNA expression, methylation, and other data on various human cancers. The mRNA expression data of 70 HNSC and 13 adjacent control samples and relevant clinical data were downloaded from the TCGA database (https://portal.gdc.cancer.gov) to further study. Clinical data information was evaluated according to the TNM staging requirements of the 8 th edition of the Joint Committee on Cancer [16].

4.5. Gene Set Enrichment Analyses. Gene Set Enrichment Analyses (GSEA) [17] is a tool for transcriptomic data that determines the enrichment of gene sets based on the correlation between gene expression data and the phenotype. First, RNA-sequencing data of 70 HNSC samples from the TCGA were divided into two groups, high and low expression, according to the TPPP3 median value of gene expression. Then, the annotated gene sets (c2.cp.KEGG.v7.0.symbols.gmt) and (c5.all.v6.2.symbols.gmt) were selected as 


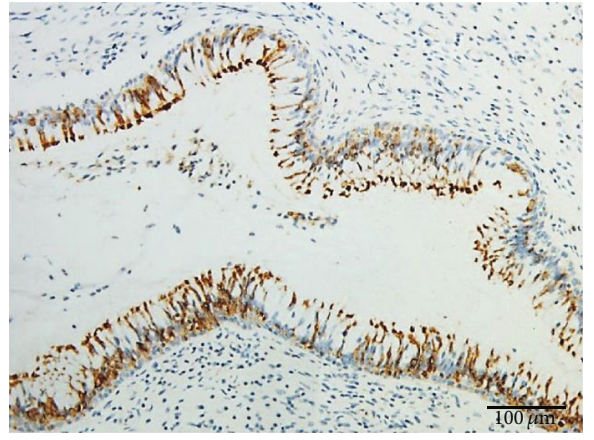

(a)

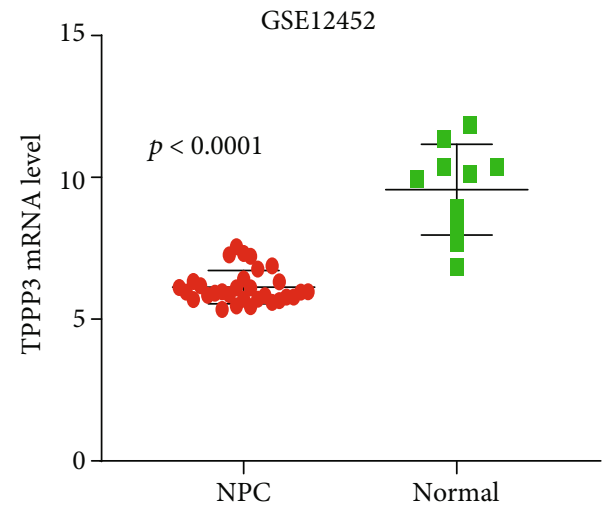

(c)

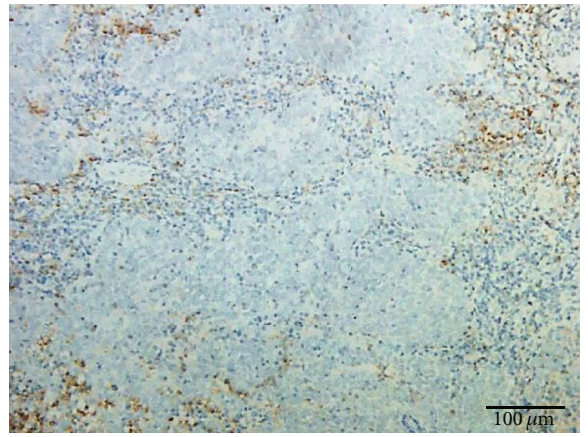

(b)

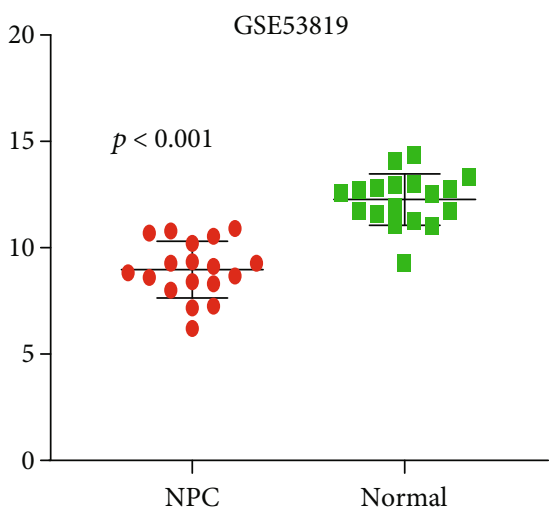

(d)

FIGURE 5: Expression of TPPP3 in nasopharyngeal carcinoma and nontumor epithelial tissue. (a) Nontumor epithelial tissue. (b) NPC tissue. (c) Analysis of TPPP3 expression levels in patients with NPC and normal tissues from GEO datasets GSE12452. $p<0.0001$. (d) Analysis of TPPP3 expression levels in patients with NPC and normal tissues from GEO datasets GSE53819. $p<0.0001$.

TABLE 1: Immunohistochemical staining showed that TPPP3 in nasopharyngeal carcinoma was lower than that in normal nasopharyngeal mucosa.

\begin{tabular}{|c|c|c|c|c|c|}
\hline Group & Positive $(+)$ & Negative (-) & Sum & Positive rate (\%) & $p$ value \\
\hline Nasopharyngeal carcinoma & 7 & 50 & 57 & 12.28 & $<0.001$ \\
\hline Normal nasopharyngeal mucosa & 27 & 3 & 30 & 90.00 & \\
\hline
\end{tabular}

reference gene sets for GSEA. Cut-off criteria were defined as $p<0.05, \mathrm{FDR}<0.05$, and enrichment score $(\mathrm{ES})>0.6$.

4.6. TIMER Website Analysis. TIMER2.0 (http://timer .cistrome.org) [18] is an interactive web server for systematically estimating the abundance of immune infiltration in tumors from the TCGA database. We performed a genetic module to analyze the correlation between TPPP3 expression and immune infiltration abundance in HNSC, including CD4+ T cells, CD8+ T cells, B cells, neutrophils, Monocyte, Macrophages, Common lymphoid progenitor, NK cell, Endothelial cell, Mast cell, Tregs, and dendritic cells, via gene modules. These genetic markers have been cited in previous researches $[19,20]$. The website provides both TIMER and CIBERSORT immune infiltration estimations.

4.7. Immunohistochemistry. NPC tissues were fixed in formalin and embedded in paraffin. First, $6 \times 8$ tissue blocks were created, and $4 \mu \mathrm{m}$ thick tissue sections were then cut and placed on glass slides. After blocking, slides were incubated at $4^{\circ} \mathrm{C}$ in a humidified chamber overnight with a monoclonal anti-TPPP3 antibody (GeneTex, USA) at a 1:200 dilution. A biotinylated secondary antibody and horseradish peroxidaselabeled avidin were subsequently applied. The diaminobenzidine method was used for visualization. The expression levels of proteins in the NPC and adjacent normal tissue sections were observed according to the staining pattern.

4.8. GEO Database Analysis. GEO (https://www.ncbi.nlm.nih .gov/geo/) is a gene expression database created and maintained by the National Center for Biotechnology Information (NCBI) in the United States. GSE12452 [21] and GSE53819 [22] datasets were obtained from the GEO database. The GSE12452 dataset contained 31 nasopharyngeal carcinomas and 10 normal healthy nasopharyngeal tissue specimens. GSE53819 contained data from 18 NPC samples and 18 noncancerous nasopharyngeal tissue samples. TPPP3mRNA 
TABLE 2: The relationship between the expression of TPPP3 and the clinicopathological features of nasopharyngeal carcinoma.

\begin{tabular}{|c|c|c|c|c|c|}
\hline Group & $n$ & Negative (-) & Positive $(+)$ & Positive proportion (\%) & $p$ value \\
\hline \multicolumn{6}{|l|}{ Gender } \\
\hline Male & 41 & 36 & 5 & 12.20 & \multirow[t]{2}{*}{$>0.05$} \\
\hline Female & 16 & 14 & 2 & 12.50 & \\
\hline \multicolumn{6}{|l|}{ Age } \\
\hline$\leq 45$ & 25 & 24 & 3 & 12.00 & \multirow[t]{2}{*}{$>0.05$} \\
\hline$>45$ & 32 & 28 & 4 & 12.50 & \\
\hline \multicolumn{6}{|c|}{ Differentiation or not } \\
\hline Differentiated & 6 & 5 & 1 & 16.67 & \multirow[t]{2}{*}{$>0.05$} \\
\hline Undifferentiated & 51 & 45 & 6 & 11.76 & \\
\hline \multicolumn{6}{|l|}{$\mathrm{T}$ stage } \\
\hline $\mathrm{T} 1, \mathrm{~T} 2$ & 21 & 19 & 2 & 9.52 & \multirow[t]{2}{*}{$>0.05$} \\
\hline T3, T4 & 36 & 31 & 5 & 13.89 & \\
\hline \multicolumn{6}{|l|}{$\mathrm{N}$ stage } \\
\hline$\geq \mathrm{N} 1$ & 52 & 46 & 6 & 11.54 & \multirow[t]{2}{*}{$>0.05$} \\
\hline N0 & 5 & 4 & 1 & 20.00 & \\
\hline \multicolumn{6}{|l|}{ M stage } \\
\hline M0 & 49 & 43 & 6 & 12.24 & \multirow[t]{2}{*}{$>0.05$} \\
\hline M1 & 8 & 7 & 1 & 12.50 & \\
\hline \multicolumn{6}{|l|}{ Clinical stage } \\
\hline I, II & 5 & 4 & 1 & 20.00 & \multirow[t]{2}{*}{$>0.05$} \\
\hline III, IV & 52 & 46 & 6 & 11.54 & \\
\hline
\end{tabular}

expression levels were compared between the tumor group and normal tissues in these two datasets.

4.9. Statistical Analysis. The mRNA expression between NPC and normal group was compared, respectively, using $t$-test. The correlation of TPPP3 expression was evaluated by Spearman's correlation and statistical significance. $p$ values $<0.05$ were considered statistically significant.

\section{Conclusions}

All in all, the TPPP3 expression is significantly reduced in HNSC, and the low expression of TPPP3 is associated with CD8 + T cell and myeloid dendritic cell immune infiltration levels. Therefore, thus, TPPP3 can serve as a potential prognostic indicator for HNSC patients and may play an important role in immune cell infiltration.

\section{Abbreviations}

TPPP3: Tubulin polymerization promoting protein family member 3

GSEA: Gene expression profiling interactive analysis

TIMER: Tumor immune estimation resource

GEPIA: Gene expression profiling interactive analysis

TCGA: The Cancer Genome Atlas

GTEx: The genotype-tissue expression

HNSC: Head and neck squamous carcinoma

MT: $\quad$ Microtubule
BLCA: Bladder urothelial carcinoma

BRCA: Breast invasive carcinoma

KICH: Kidney chromophobe

KIRC: Kidney renal papillary cell carcinoma

LUAD: Lung adenocarcinoma

LUSC: Lung squamous cell carcinoma

CHOL: Cholangiocarcinoma

NPC: Nasopharyngeal carcinoma

WHO: The World Health Organization

OS: $\quad$ Overall survival

DFS: Disease-free survival.

\section{Data Availability}

The data used in this study are from open public databases, and how to obtain them has been explained in the manuscript.

\section{Disclosure}

The funders had no role in the design of the study; in the collection, analyses, or interpretation of data; in the writing of the manuscript; or in the decision to publish the results.

\section{Conflicts of Interest}

The authors declare no conflict of interest. 


\section{Authors' Contributions}

Z.Y. and W.M. did the conceptualization; Z.Y. and X.L. did the methodology; Z.Y., J.L., and Q.S. was assigned on the software; Z.Y., X.L., J.L., Q.S., Y.Q., Z.Z., L.Z., and W.M. did the validation; Z.Y did the formal analysis; W.M. was assigned on the resources; X.L. did the data curation; Z.Y. wrote the original draft preparation; Z.Y. and Q.S. did the writing, review, and editing; Z.Y. did the visualization; Y.Q. supervised; W.M. did the project administration; Z.Y. and W.M. was assigned on the funding acquisition. All authors have read and agreed to the published version of the manuscript.

\section{Acknowledgments}

This research was funded by the Natural Science Foundation of Guangxi, China, grant number 2018GXNSFAA281055, and the Guangxi Zhuang Autonomous Region Health Committee self-funded scientific research, grant number Z20190841.

\section{References}

[1] D. Hanahan and R. A. Weinberg, "Hallmarks of cancer: the next generation," Cell, vol. 144, no. 5, pp. 646-674, 2011.

[2] N. Raab-Traub, "Epstein-Barr virus in the pathogenesis of NPC," Seminars in Cancer Biology, vol. 12, no. 6, pp. 431441, 2002.

[3] X. Yang, "Evaluation of risk factors for nasopharyngeal carcinoma in high-risk nasopharyngeal carcinoma families in Taiwan," Cancer Epidemiology, Biomarkers \& Prevention, vol. 14, no. 4, pp. 900-905, 2005.

[4] L.-F. Hu, Q.-H. Qiu, S.-M. Fu et al., “A genome-wide scan suggests a susceptibility locus on $5 \mathrm{p} 13$ for nasopharyngeal carcinoma," European Journal of Human Genetics, vol. 16, no. 3, pp. 343-349, 2008.

[5] S. K. Yong, T. C. Ha, M. C. R. Yeo, V. Gaborieau, J. D. McKay, and J. Wee, "Associations of lifestyle and diet with the risk of nasopharyngeal carcinoma in Singapore: a case-control study," Chinese Journal of Cancer, vol. 36, no. 1, 2017.

[6] O. Vincze, N. Tökési, J. Oláh et al., “Tubulin polymerization promoting proteins (TPPPs): members of a new family with distinct structures and functions," Biochemistry, vol. 45, no. 46, pp. 13818-13826, 2006.

[7] A. L. Parker, W. S. Teo, J. A. McCarroll, and M. Kavallaris, “An emerging role for tubulin isotypes in modulating cancer biology and chemotherapy resistance," International Journal of Molecular Sciences, vol. 18, no. 7, 2017.

[8] W. Zhou, X. Wang, L. Li et al., "Depletion of tubulin polymerization promoting protein family member 3 suppresses HeLa cell proliferation," Molecular and Cellular Biochemistry, vol. 333, no. 1-2, pp. 91-98, 2010.

[9] D. A. Oble, R. Loewe, P. Yu, and M. C. Mihm, "Focus on TILs: prognostic significance of tumor infiltrating lymphocytes in human melanoma," Cancer Immunity, vol. 9, no. 1, 2009.

[10] S. J. Dovedi, G. Lipowska-Bhalla, S. A. Beers et al., "Antitumor efficacy of radiation plus immunotherapy depends upon dendritic cell activation of effector CD8+ T cells," Cancer Immunology Research, vol. 4, no. 7, pp. 621-630, 2016.
[11] C. S. Garris, S. P. Arlauckas, R. H. Kohler et al., "Successful anti-PD-1 cancer immunotherapy requires $\mathrm{T}$ cell-dendritic cell crosstalk involving the cytokines IFN- $\gamma$ and IL-12," Immunity, vol. 49, no. 6, pp. 1148-1161.e7, 2018.

[12] Y. Li, M. Bai, Y. Xu, W. Zhao, N. Liu, and J. Yu, “TPPP3 promotes cell proliferation, invasion and tumor metastasis via STAT3/Twist1 pathway in non-small-cell lung carcinoma," Cellular Physiology and Biochemistry, vol. 50, no. 5, pp. 2004-2016, 2018.

[13] K. Ye, Y. Li, W. Zhao et al., "Knockdown of tubulin polymerization promoting protein family member 3 inhibits cell proliferation and invasion in human colorectal cancer," Journal of Cancer, vol. 8, no. 10, pp. 1750-1758, 2017.

[14] D. R. Rhodes, S. Kalyana-Sundaram, V. Mahavisno et al., "Oncomine 3.0: genes, pathways, and networks in a collection of 18,000 cancer gene expression profiles," Neoplasia, vol. 9, no. 2, pp. 166-180, 2007.

[15] Z. Tang, C. Li, B. Kang, G. Gao, C. Li, and Z. Zhang, "GEPIA: a web server for cancer and normal gene expression profiling and interactive analyses," Nucleic Acids Research, vol. 45, no. W1, pp. W98-W102, 2017.

[16] M. B. Amin, S. Edge, F. Greene et al., AJCC Cancer Staging Manual, Springer International Publishing, 8th edition, 2017.

[17] A. Subramanian, P. Tamayo, V. K. Mootha et al., "Gene set enrichment analysis: a knowledge-based approach for interpreting genome-wide expression profiles," Proceedings of the National Academy of Sciences of the United States of America, vol. 102, no. 43, pp. 15545-15550, 2005.

[18] T. Li, J. Fan, B. Wang et al., "TIMER: a web server for comprehensive analysis of tumor-infiltrating immune cells," Cancer Research, vol. 77, no. 21, pp. e108-e110, 2017.

[19] N. O. Siemers, J. L. Holloway, H. Chang et al., "Genome-wide association analysis identifies genetic correlates of immune infiltrates in solid tumors," PLoS One, vol. 12, no. 7, article e0179726, 2017.

[20] M. D. Iglesia, J. S. Parker, K. A. Hoadley, J. S. Serody, C. M. Perou, and B. G. Vincent, "Genomic analysis of immune cell infiltrates across 11 tumor types," Journal of the National Cancer Institute, vol. 108, no. 11, 2016.

[21] L. E. Dodd, S. Sengupta, I. H. Chen et al., "Genes involved in DNA repair and nitrosamine metabolism and those located on chromosome $14 \mathrm{q} 32$ are dysregulated in nasopharyngeal carcinoma," Cancer Epidemiology, Biomarkers \& Prevention, vol. 15, no. 11, pp. 2216-2225, 2006.

[22] Y. N. Bao, X. Cao, D. H. Luo et al., "Urokinase-type plasminogen activator receptor signaling is critical in nasopharyngeal carcinoma cell growth and metastasis," Cell Cycle, vol. 13, no. 12, pp. 1958-1969, 2014. 